\title{
THE GLOBULAR CLUSTER SYSTEM OF THE MILKY WAY: ACCRETION IN A COSMOLOGICAL CONTEXT
}

\author{
Stefan C. Keller, Dougal Mackey, and Gary S. Da Costa \\ RSAA, Australian National University, Weston Creek, ACT 2611, Australia \\ Received 2011 January 24; accepted 2011 September 8; published 2011 December 13
}

\begin{abstract}
We examine the significance of a planar arrangement in the spatial distribution of the Milky Way (MW) globular clusters (GCs). We find that, when separated on the basis of horizontal branch morphology and metallicity, the outermost canonical young halo (YH) GC sample (at galactocentric radii in excess of $10 \mathrm{kpc}$ ) exhibits an anisotropic distribution that may be equated to a plane $(24 \pm 4) \mathrm{kpc}$ thick (rms) and inclined at $8^{\circ} \pm 5^{\circ}$ to the polar axis of the MW disk. To quantify the significance of this plane we determine the fraction of times that an isotropic distribution replicates the observed distribution in Monte Carlo trials. The plane is found to remain significant at the $>95 \%$ level outside a galactocentric radius of $10 \mathrm{kpc}$, inside this radius the spatial distribution is apparently isotropic. In contrast, the spatial distribution of the old halo sample outside $10 \mathrm{kpc}$ is well matched by an isotropic distribution. The plane described by the outer YH GCs is indistinguishable in orientation from that presented by the satellite galaxies of the MW. Simulations have shown that the planar arrangement of satellites can arise as filaments of the surrounding large-scale structure feed into the MW's potential. We therefore propose that our results are direct observational evidence for the accreted origin of the outer YH GC population. This conclusion confirms numerous lines of evidence that have similarly indicated an accreted origin for this set of clusters from the inferred cluster properties.
\end{abstract}

Key words: Galaxy: evolution - globular clusters: general

Online-only material: color figures

\section{INTRODUCTION}

It has long been noted that the most luminous satellite galaxies of the Milky Way (MW) exhibit an asymmetric spatial distribution as seen on the sky (Lynden-Bell 1976, 1982; Kunkel \& Demers 1976). Kroupa et al. (2005) demonstrated that the spatial distribution of the 11 most luminous MW satellites is inconsistent with an isotropic distribution in $99.5 \%$ of random realizations. Rather, these satellite galaxies describe a planar structure that extends $254 \mathrm{kpc}$ from the MW and possesses a scale height of $\sim 20 \mathrm{kpc}$; the MW plane of satellites (PoS; Metz et al. 2007, 2008). ${ }^{1}$ Metz et al. (2009a) extend this work to include the newly discovered ultra-faint dwarf galaxies with minimal change to the determined spatial parameters of this plane.

The $\mathrm{PoS}$ so described is seen to be within $\sim 8^{\circ}$ of polar to the rotation axis of the MW. The Andromeda galaxy (M31) is the only other extensive satellite system for which we can describe the three-dimensional structure with meaningful accuracy. A similar degree of planarity is evident around M31 (Koch \& Grebel 2006). Metz et al. (2009a) updated this analysis to include increased numbers of M31 satellites and found that the PoS around M31 is inclined at $59^{\circ}$ with respect to the disk (it should be noted that the number of M31 satellites has increased by $\sim 50 \%$ since the work of Metz et al. (2009b) however; see Martin et al. 2009 and Richardson et al. 2011).

The configuration of satellites about their host galaxy rose to prominence with the study of Holmberg (1969), who proposed that satellites are preferentially found near the poles of the host galaxy disk (the "Holmberg effect"). Subsequent studies based

\footnotetext{
1 We do not use the term "disk of satellites" of these authors, as the term "disk" implies a circular or rotationally supported configuration in three dimensions that is not constrained in our discussion to follow.
}

on extensive redshift surveys have not confirmed this effect (Sales \& Lambas 2009; Bailin et al. 2008). When averaged over all host-satellite pairs, the satellites of relatively isolated galaxies (akin to the MW and M31) have a tendency to be found near the major axis of their host (Brainerd 2005; Agustsson \& Brainerd 2010). The findings that the satellite planes of the MW and M31 are highly inclined are therefore unexpected.

Recently, Kroupa et al. (2010) have argued that the existence of the MW PoS is one of a number of critical challenges for Lambda cold dark matter $(\Lambda \mathrm{CDM})$ cosmology. Kroupa et al. propose that the MW satellite system is a system of tidal dwarf galaxies that resulted from a major "wet" merger that gave rise to the galactic bulge. However, Libeskind et al. (2011) and Lovell et al. (2011) contend that the observed spatial distribution and possible rotating configuration of the MW PoS is not an insurmountable problem for $\Lambda \mathrm{CDM}$ and can arise naturally from hierarchical structure growth. In their simulations the MW satellite system properties can be frequently matched when accretion occurs preferentially along filaments of cosmic DM structure. Issues concerning cosmogony aside, the MW PoS is accepted to comprise a system that has resulted from accretion to the MW halo.

It was the seminal work of Searle \& Zinn (1978) that utilized the horizontal branch (HB) morphology of the outer halo globular clusters (GCs), together with the absence of an abundance gradient within the outer halo GCs, to propose that accretion onto the early MW was substantial, and in the case of the outer halo, the dominant formation mechanism. Subsequently, Zinn $(1985,1993)$ and, more recently, Mackey \& Gilmore (2004) present a reanalysis of the Searle \& Zinn result. In these previous studies, the MW GC population is split on the basis of HB morphology and metallicity into three subgroups: an old halo $(\mathrm{OH}) \mathrm{GC}$ population which has blue $\mathrm{HB}$ morphology at given $[\mathrm{Fe} / \mathrm{H}]$ and ages $\sim 13 \mathrm{Gyr}$; disk/bulge 
(DB) GCs of relatively high metallicity and exclusively red HB morphology; and a "young halo" (YH) population of GCs with comparatively red $\mathrm{HB}$ morphology at given $[\mathrm{Fe} / \mathrm{H}]$.

The YH GCs, as defined by Mackey \& Gilmore, span a distance range far in excess of the other populations: from $2-120 \mathrm{kpc}$, as distinct from, for instance, the $\mathrm{OH}$ population where the majority reside within $30 \mathrm{kpc}$. In terms of kinematics, the YH GC system shows no evidence for net rotation, while the old GC system shows prograde rotation. Mackey \& Gilmore demonstrate the young system resembles the GC systems of the Large and Small Magellanic Clouds (LMC and SMC), Fornax and Sagittarius dwarf galaxies in terms of metallicity range, age, and $\mathrm{HB}$ morphology. Furthermore, the YH GC population possesses generally more extended structures than their $\mathrm{OH}$ counterparts. The distribution of core radii for the $\mathrm{YH}$ system is seen to match that of the above-mentioned four external dwarf galaxies, and distinct from the core and half-light radius distribution of the $\mathrm{OH}$ GC subsystem to high significance (Mackey \& Gilmore 2004). Based on these above similarities between the YH GC subsystem and the external GC systems, Mackey \& Gilmore (2004) propose that the YH GCs are of extragalactic origin (in accordance with the result of Searle \& Zinn 1978), whereas the majority (but not all) of the $\mathrm{OH}$ and DB GCs are proposed to have formed in-situ under some form of dissipative collapse as originally envisaged by Eggen et al. (1962).

The study of Yoon \& Lee (2002) presented evidence for a planar arrangement of a set of seven Galactic GCs (GGCs). These seven clusters were selected based on the RR Lyrae Oosterhoff dichotomy (as Oosterhoff Type-2 clusters) and metallicity (the lowest metallicity clusters in the Type- 2 group). Yoon \& Lee (2002) propose that these clusters have been accreted from an external galaxy. However, as pointed out by Catelan (2009), the present-day MW satellites possess GC systems that do not match the Oosterhoff dichotomy and the distribution of GGCs in the HB morphology-metallicity plane. This discrepancy with present-day satellite galaxies aside, the studies of Marín-Franch et al. (2009) and Dotter et al. (2010, 2011) show that the age-metallicity relation of the GGCs possesses two branches-one with near constant old age of $\sim 13$ Gyr and another branch to significantly younger ages with increased $[\mathrm{Fe} / \mathrm{H}]$. The younger branch of GGCs is found to be dominated by GCs associated with the Sagittarius and Canis Major dwarf galaxies. This leads Forbes \& Bridges (2010) to conclude that approximately one-quarter of the GGC system has resulted from accretion.

In this study, we show that the YH GCs of the MW (as defined by a combination of age estimators) are confined to a plane that is indistinguishable from the MW PoS. The YH GCs act as tracers of the dwarf galaxies that have been disrupted in the formation of the outer halo. The consistency in spatial alignment of early accretion events to the alignment of current satellites suggests a preferred orientation for accretion from the local large-scale structure (LSS) that has remained consistent for a large fraction of a Hubble time.

\section{PARTITION OF THE GLOBULAR CLUSTER POPULATION BY RELATIVE CLUSTER AGE}

When excellent self-consistent photometry exists, such as in the study of Marín-Franch et al. (2009), it is possible to derive accurate relative ages even amongst clusters of advanced age. These authors compare the luminosity of the main-sequence turnoff to that of the lower main sequence and hence express the

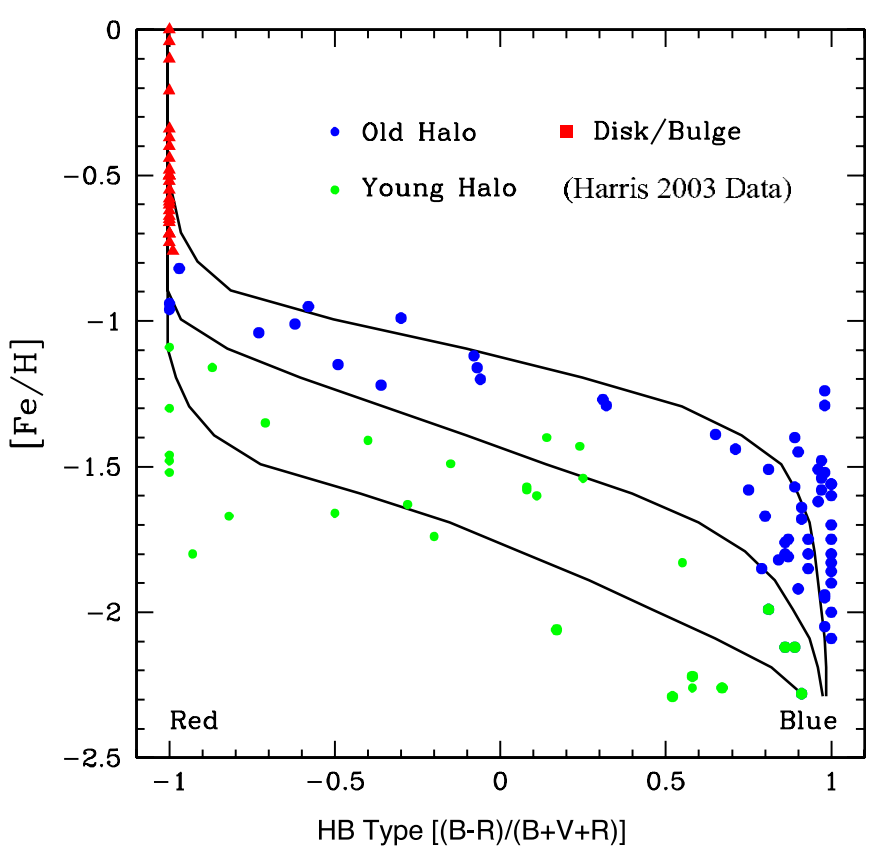

Figure 1. HB-type vs. metallicity diagram for the 114 Galactic globular clusters with suitable measurements in the 2003 and 2010 versions of the Harris (1996) catalog. The clusters have been divided into three subsystems, as labeled, according to the criteria set out in the text. The overplotted isochrones are from Rey et al. (2001). The two lower isochrones are, respectively, 1.1 Gyr and 2.2 Gyr younger than the top isochrone.

(A color version of this figure is available in the online journal.)

relative age for a sample of 64 nearby GCs. Marín-Franch et al. find evidence for two groups of GCs: an old GC population with an age dispersion of $\sim 5 \%$ and no age-metallicity relation, and a young GC population with an age-metallicity relation similar to that defined by the GCs associated with the Sagittarius dwarf galaxy.

The age determination method of Zinn (1993) relies on a relation between age, metallicity, and $\mathrm{HB}$ morphology to resolve relative cluster age differences. Zinn defined a fiducial sequence for inner halo clusters $\left(R_{\mathrm{gc}}<6 \mathrm{kpc}\right)$ in a HB-type ${ }^{2}$ against $[\mathrm{Fe} / \mathrm{H}]$ diagram and then measured the difference between the HB-type of a given cluster and that of the fiducial sequence. Those with a difference in HB-type $>-0.4$ were classified by Zinn (1993) as young clusters, the remainder old clusters. Mackey \& Gilmore (2004) has revisited the issue of relative age partition. Rather than using spatial information in the derivation of their fiducial these authors utilize the model evolutionary isochrones of Rey et al. (2001). The isochrone corresponding to the canonical ancient GC population traces of those clusters with the bluest HB-type at a given $[\mathrm{Fe} / \mathrm{H}]$ (see Figure 1). In the study of Mackey \& Gilmore (2004), the YH GC population is defined as those clusters with a difference in HB-type in excess of -0.3 from that of the fiducial at a corresponding $[\mathrm{Fe} / \mathrm{H}]$. This corresponds to a minimum age difference of around -0.6 Gyr at HB-type $=0$. It should be noted that there is an important limitation in the application of this technique for estimating the relative ages between clusters. At the extremes of HB-type the isochrones become degenerate within observational uncertainties. We note that parameters other than age can also be responsible for the location of a cluster in these groupings.

\footnotetext{
$2 \operatorname{HBR}=\left[\frac{(B-R)}{(B+V+R)}\right]$, where $B$ is the number of stars bluer than the instability strip, $V$ is the number of stars within the instability strip, and $R$ is the number of stars to the red of the instability strip.
} 
For example, in the case of NGC 2808 the extreme blue HB morphology is likely driven by He abundance variations.

In the study of Yoon \& Lee (2002), relative age differences of around $1 \mathrm{Gyr}$ are inferred from an examination of the mean period of type-ab RR Lyraes $\left(\left\langle P_{\mathrm{ab}}\right\rangle\right)$ with cluster metallicity. Oosterhoff Group I clusters possess $\left\langle P_{\mathrm{ab}}\right\rangle \sim 0.55$ days and are more metal-rich than those of Group II with $\left\langle P_{\mathrm{ab}}\right\rangle \sim 0.65$ days. Yoon \& Lee (2002) demonstrate that Group II clusters may be further split into "old" and "young" clusters (Groups II-a and II-b, respectively). The benefit of this method is that we can continue our classification to the blue extreme of HB-type. A limitation on the method is that the $\left\langle P_{\mathrm{ab}}\right\rangle$ of many clusters is either limited by intrinsically low RR-ab numbers or the absence of studies in the literature (see Catelan 2009).

Our partitioning of the GGC population will rely on a combination of the latter two methods. In the regime where both methods are viable (that is HB-type $<0.8$, and $\left\langle P_{\mathrm{ab}}\right\rangle>$ 0.60 days) there are six objects in common: both methods classify five as "young," the exception is the GC Rup 106 for which the $\left(\left\langle P_{\mathrm{ab}}\right\rangle,[\mathrm{Fe} / \mathrm{H}]\right)$ method would suggest "old" and the (HB-type, $[\mathrm{Fe} / \mathrm{H}]$ ) method would suggest "young." Examination of the main-sequence turn-off (MSTO) in the color-magnitude diagram (CMD) demonstrates that it is indeed younger than most GCs (Marín-Franch et al. 2009). For the analysis that follows we will use the following criteria:

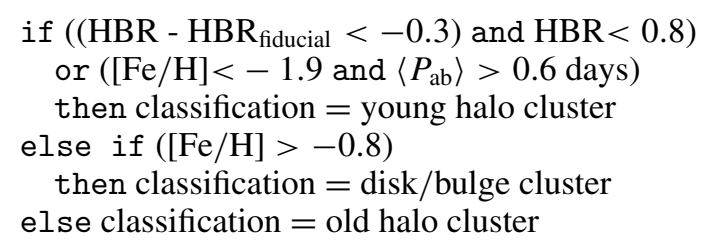

Graphically, our partition of the GC population can be seen in Figure 1. We take the $[\mathrm{Fe} / \mathrm{H}]$, and in the analysis to follow, the distances and positions of GCs from the compilation of Harris (1996, 2010 edition). The HBR is taken from the 2003 edition of the Harris catalog. Koposov 1 and 2 are not included here as they lack sufficient data to constrain their HB-type. The pulsation properties of the clusters are taken from the compilation of Catelan (2009). The three GC subgroups YH, $\mathrm{OH}$, and $\mathrm{DB}$, as defined above, contain 30,56 , and 28 clusters, respectively. Compared to the work of Mackey \& Gilmore (2004), our selection criteria sees the clusters NGC 5024, 6341, and 7099 included in our YH grouping on the basis of the pulsation properties of their RR Lyrae populations, whereas these clusters were previously assigned to the $\mathrm{OH}$ grouping.

\section{THE SPATIAL DISTRIBUTION OF GLOBULAR CLUSTERS}

We now investigate how isotropic the spatial distributions of the three GC populations are. We utilize the method described in detail in Metz et al. (2007). In brief, we utilize a leastsquares technique to define the plane that best matches the spatial distribution and determine the normal vector to this plane. Since the least-squares method is unweighted, we account for distance uncertainties by repeat realizations of the system with objects randomly shifted along their lines of sight according to a normal distribution function in which the variance matches distance uncertainty to each object. This provides an estimation of the robustness of a disk-like population. If the GC sample is not distributed in a well-defined plane, then repeat realizations of the sample to account for distance uncertainties will show a large scatter in the orientation of the normal vector. A large number of realizations $\left(10^{4}\right)$ of the system are made.

This results in a set of $m$ unit normal vectors $\hat{\mathbf{n}}$ for which $\left(\hat{x}_{i}, \hat{y}_{i}, \hat{z}_{i}\right)$ are the Cartesian components. We then define the matrix $\mathbf{M}$ as

$$
\mathbf{M}=\left(\begin{array}{ccc}
\hat{x}_{1} & \hat{y}_{1} & \hat{z}_{1} \\
\hat{x}_{2} & \hat{y}_{2} & \hat{z}_{2} \\
\vdots & \vdots & \vdots \\
\hat{x}_{m} & \hat{y}_{m} & \hat{z}_{m}
\end{array}\right)
$$

and perform an eigenvalue analysis of the matrix $\mathbf{T}=\mathbf{M}^{\mathrm{T}} \mathbf{M}$. The eigenvector that corresponds to the largest eigenvalue of $\mathbf{T}$ is the principal axis of the set of input vectors. This principal axis defines the orientation of spatial anisotropy in the input GC sample. There are three eigenvalues of $\mathbf{T}, \tau_{1}<\tau_{2}<\tau_{3}$, where $\tau_{3}$ is the eigenvalue of the principal axis. In the case of a uniform spherical distribution $\tau_{1}=\tau_{2}=\tau_{3}=1 / 3$. Following the formalism of Fisher et al. (1987, their Section 3.4), the following parameters derived from the eigenvalues provide us with a measure of how isotropic the distribution is. We define a shape parameter $\gamma$,

$$
\gamma=\frac{\ln \left(\tau_{3} / \tau_{2}\right)}{\ln \left(\tau_{2} / \tau_{1}\right)},
$$

and a strength parameter $\zeta$,

$$
\zeta=\ln \left(\tau_{3} / \tau_{1}\right)
$$

The parameters $\gamma$ and $\zeta$ enable the classification of the spherical distribution of normal vectors. For example, a high value of $\gamma$ (say 3 or more) indicates a clustered distribution of normals on the sky. When combined with a high value for $\zeta$, this indicates a strongly condensed distribution. A low value for $\gamma$ indicates the presence of a girdled distribution, i.e., a distribution that forms a band around the sky. $\zeta$ then describes how strongly confined the distribution is to this girdle.

We now consider the distributions of our three GC groups. We compare the spherical distribution of the normal vectors to the best-fitting plane with those arising from simulations of an isotropically distributed model GC population. The isotropic GC model is constructed from a density power law of index -3.5 , which is appropriate for the overall MW GC population (Parmentier \& Grebel 2005). In galactic coordinates this model population is uniformly distributed. The number of simulated GCs is matched to the observed number of GCs in each age group.

The results of this comparison are seen in Figure 2. Here the shaded regions show the probability distribution function of the isotropic sample (realized $10^{4}$ times). The star symbol in each panel shows the peak of the probability density function for the observed GC sample (simulated $10^{4}$ times to account for the distance uncertainty to each object). In the case of the $\mathrm{OH}$ and $\mathrm{DB}$ GCs, the spatial distribution of the observed sample is indistinguishable from that of an isotropic distribution of the same size. With no restriction on the inner radius of the YH GC sample, there is a suggestion from the offset of the probability density function peaks that the YH sample is less well described by an isotropic parent distribution (see Figure 2, lower right panel).

To quantify the hypothesis that the spatial distribution of the observed sample is derived from an isotropic parent distribution, we determine the contour lines seen in Figure 2. These contour lines enclose regions of the diagram which contain a given percentage of the isotropic sample. The percentage of times 


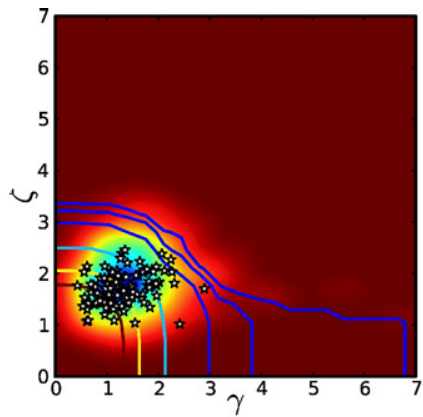

Disk-Bulge GCs

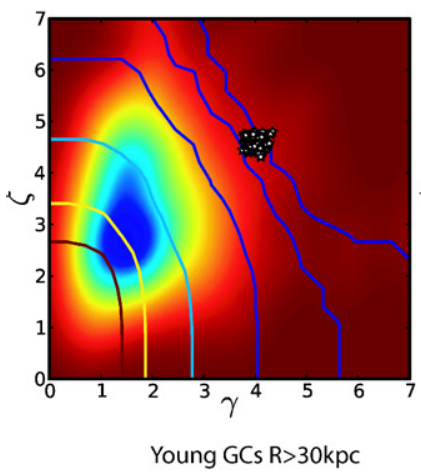

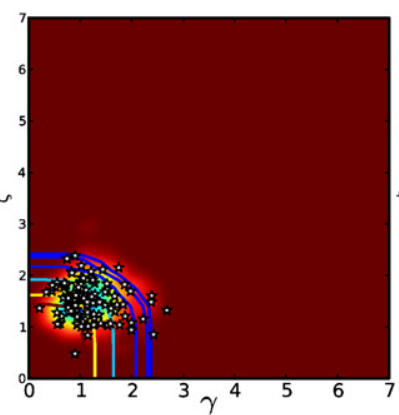

Old Halo GCs

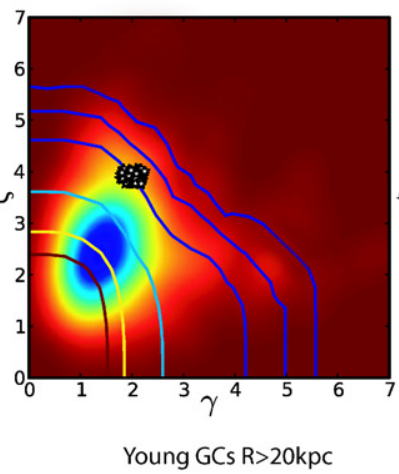

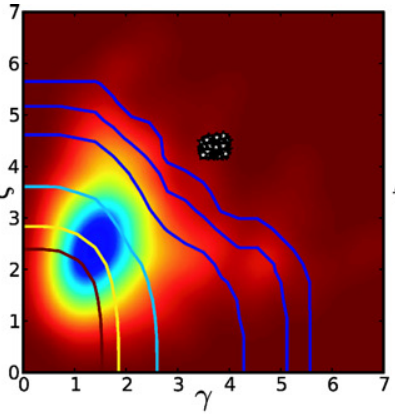

Classical LG

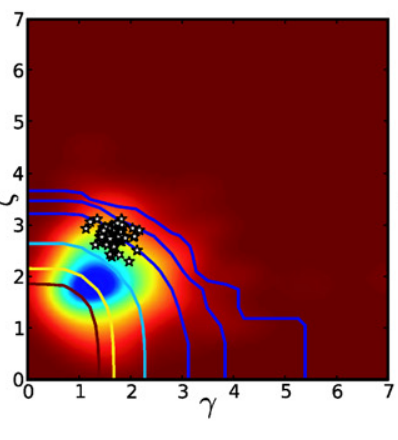

Young GCs R>10kpc

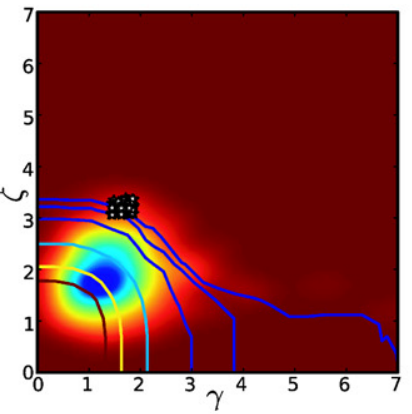

Young GCs+Classical LG+UFDs

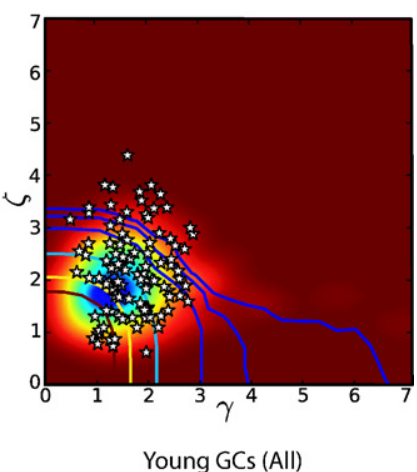

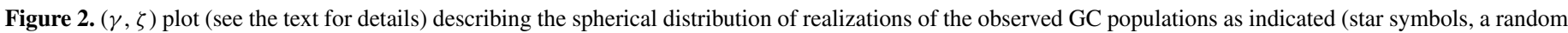

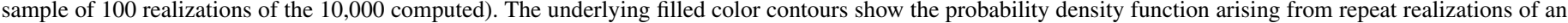

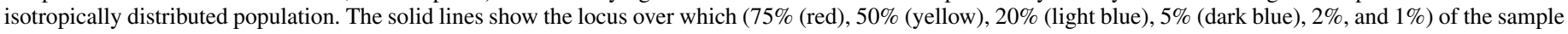
is of greater $\gamma$ and $\zeta$.

(A color version of this figure is available in the online journal.)

Table 1

The Results of Our Determination of Best-fitting Plane to the Distribution of Objects as Described in Section 3

\begin{tabular}{|c|c|c|c|c|c|c|}
\hline $\begin{array}{l}\text { GC } \\
\text { Group }\end{array}$ & $\begin{array}{l}R_{\mathrm{GC}} \\
(\mathrm{kpc})\end{array}$ & $\begin{array}{l}\text { Number } \\
\text { Clusters }\end{array}$ & $\begin{array}{c}\ell \\
\left({ }^{\circ}\right)\end{array}$ & $\begin{array}{c}b \\
\left({ }^{\circ}\right)\end{array}$ & $\begin{array}{c}\text { rms Thickness }{ }^{\mathrm{a}} \\
(\mathrm{kpc})\end{array}$ & $\begin{array}{c}\text { Significance }^{\mathrm{b}} \\
(\%)\end{array}$ \\
\hline $\mathrm{OH}$ & All & 56 & $\ldots$ & $\ldots$ & $\ldots$ & 54 \\
\hline $\mathrm{OH}$ & $>20$ & 6 & $\ldots$ & $\ldots$ & $\ldots$ & 56 \\
\hline DB & All & 28 & $\ldots$ & $\ldots$ & $\ldots$ & 53 \\
\hline YH & All & 30 & $144 \pm 6$ & $-13 \pm 5$ & $28 \pm 5$ & 64 \\
\hline YH & $>10$ & 23 & $156 \pm 6$ & $-8 \pm 5$ & $24 \pm 4$ & 95 \\
\hline YH & $>20$ & 10 & $169 \pm 5$ & $-9 \pm 6$ & $23.2 \pm 2.7$ & 95 \\
\hline $\mathrm{YH}$ & $>30$ & 7 & $162 \pm 5$ & $-8 \pm 7$ & $18.5 \pm 2.3$ & 99.2 \\
\hline $\mathrm{CS}^{\mathrm{c}}$ & All & 11 & $156.8 \pm 2.7$ & $-11.6 \pm 2.9$ & $19.3 \pm 2.1$ & 99.1 \\
\hline $\mathrm{CS}+\mathrm{UFD}^{\mathrm{d}}$ & All & 21 & $168 \pm 4$ & $-13 \pm 3$ & $25 \pm 3$ & 98 \\
\hline $\mathrm{YLO}^{\mathrm{e}}$ & All & 7 & $183 \pm 15$ & $-3 \pm 9$ & $19 \pm 4$ & 96 \\
\hline
\end{tabular}

Notes.

${ }^{\text {a }}$ Calculated from repeat random simulations of the distance uncertainties for each object. See the text for details.

${ }^{b}$ Percentage of times the observed result is not replicated drawing from an isotropic population.

c The 11 most luminous ("classical") satellite galaxies of the Milky Way.

d As above with the inclusion of 10 low-luminosity dwarf galaxies. See the text for details.

e The cluster sample of Yoon \& Lee (2002).

the centroid of the observed probability density function is replicated by the isotropic simulated sample for each GC group is given in Table 1.

The validity of a spatially isotropic parent population does not change significantly for the $\mathrm{OH}$ and $\mathrm{DB}$ GC groups as we constrain the galactocentric radius of the sample. In the case of the $\mathrm{OH}$ GC group, 19 clusters are of $R_{\mathrm{GC}}>10 \mathrm{kpc}$ and six are $R_{\mathrm{GC}}>20 \mathrm{kpc}$; however, these samples are consistent with an isotropic parent distribution in $54 \%$ and $56 \%$ of trials, respectively. In the case of the YH GC group, however, the hypothesis of a spatially isotropic parent distribution becomes increasingly unlikely as we restrict the sample to more distant objects. As seen in the $\gamma-\zeta$ plane (Figure 2) the YH GCs are increasingly located at larger $\gamma$ and $\zeta$ with increasing galactocentric distance. This is indicative that the spherical distribution of the normal vectors to the best-fitting plane for the population is becoming increasingly confined to a preferred direction. That is to say, the YH GCs are seen to be increasingly confined to a planar alignment as we progress to larger galactocentric distances. In Table 1 we summarize our findings regarding the orientation and rms thickness of the plane that best matches to the observed sample.

We now turn our attention to the spatial distribution of the satellite galaxies of the MW. Figure 2 shows the corresponding 


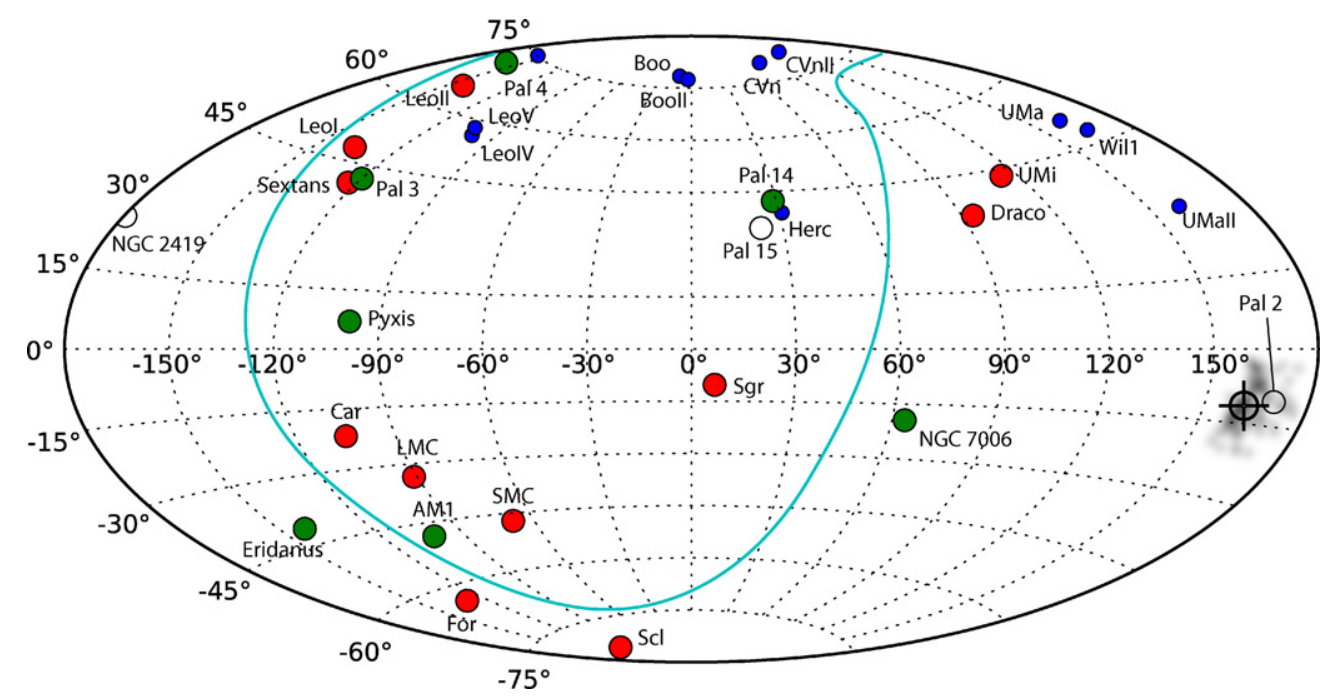

Figure 3. Plane of satellites (solid line; derived from the young halo GC population of galactocentric distance greater than $10 \mathrm{kpc}$; see Table 1) seen on an Aitoff projection in Galactic coordinates. Red, green filled circles represent the 11 "classical" luminous satellite galaxies of the Milky Way and the YH globular clusters $\left(R_{\mathrm{GC}}>30 \mathrm{kpc}\right)$, respectively. The open circles show the positions of outer $\mathrm{OH}$ clusters. The smaller blue points show the positions of the "ultra-faint" dwarf satellites. Note that this figure is a two dimensional projection of the three-dimensional distribution of satellites. For this reason the distance from the line (which is the plane of satellites seen at infinity) does not correspond to distance from the plane. The region of gray scale on the right of the figure shows the probability density function of the normal to this plane from repeated random realizations of the set of objects to account for uncertainties in their distance. The cross hair denotes the highest likelihood position of this normal to the plane.

(A color version of this figure is available in the online journal.)

$\gamma-\zeta$ plot for the 11 most luminous satellites of the MW (i.e., identical to Metz et al. 2007). The significance, orientation, and thickness of a plane to the classical dwarf galaxy sample are given in Table 1. Our results may be compared to those of Metz et al. (2007) who find $\ell=158.2, b=-11.9$, and an rms thickness, $\Delta=32.6 \mathrm{kpc}$. Figure 2 (center) shows our findings with the inclusion of 10 recently discovered lowluminosity MW satellites (replicating the sample of Metz et al. 2009b). Here our results may be compared to those of Metz et al. (2009b) who find $\ell=159.7 \pm 2.3, b=$ $-6.8 \pm 2.3$, and $\Delta=24.9 \pm 1.1 \mathrm{kpc}$. It may be argued, on the basis of the results of Besla et al. (2010), that the Magellanic Clouds (MCs) be considered separately since their proper motions suggest they are on their first passage by the MW. On the other hand, since the MCs lie on a plane common to the remaining nine "classical" satellites, we propose that if the MCs are on their first infall that they are further evidence in favor of preferred accretion along a common plane. In both of the above samples we recover the significance, orientation, and thickness of the planar distributions discussed in the literature.

It is apparent from Table 1 that the orientation and thickness of the plane that describes the spatial distribution of the $\mathrm{YH}$ GCs at $R_{\mathrm{GC}}>10 \mathrm{kpc}$ are indistinguishable from those of the classical MW dwarf galaxies and the recently discovered "ultrafaint" dwarf galaxies. For this reason we combine these three samples to define a common PoS.

Figure 3 shows the spatial distribution on the sky of the classes of object we have considered. The PoS, as defined above, is shown as the solid line. The gray-scale probability density function seen at $\ell \sim 156^{\circ}$ shows the positions of the normal to this plane in $10^{4}$ random realizations of the input catalog distance uncertainties.

The top left panel of Figure 4 shows a section through the vicinity of the MW as seen at a viewing angle edge-on to our derived PoS. We note that the OH GC NGC 2419 (the open diamond in the figure) resides well away from this plane at a distance of $83 \mathrm{kpc}, 3.4$ times the thickness of the PoS. There is increasing evidence that NGC 2419 is an accreted object. Specifically, Cohen et al. (2010) argue that due to its internal $[\mathrm{Fe} / \mathrm{H}]$ spread this cluster is the former nucleus (or nuclear star cluster) of a disrupted dwarf. Potentially therefore NGC 2419 had been accreted away from the plane apparently preferred by the vast majority of the MW's accreted objects.

Our findings reinforce those of Yoon \& Lee (2002) from a sample of GCs restricted to $R_{\mathrm{GC}}<20 \mathrm{kpc}$. In that study, these authors isolated a set of YH clusters based on metallicity and RR Lyrae pulsation period (as discussed in Section 2). Their set of seven young GCs (their "group II-b") describes a plane orientated toward $\ell=183^{\circ} \pm 15^{\circ}, b=-3^{\circ} \pm 9^{\circ}$ (at $96 \%$ significance compared to an isotropic distribution), which is consistent with the PoS derived here. The PoS is therefore persistent in the spatial distribution of the MW's YH GCs to the inner galaxy.

Although we here use YH GCs as a tracer of accreted systems, it is expected that accreted systems will deposit both $\mathrm{YH}$ and $\mathrm{OH}$ clusters. As pointed out by Da Costa \& Armandroff (1995), the Sgr dwarf is contributing to both groups. Furthermore, Mackey \& Gilmore (2004) propose that 15\%-17\% of the $\mathrm{OH}$ GCs were contributed via accretions. The fact that our analysis has not shown any radial anisotropy in the distribution of $\mathrm{OH}$ GCs is perhaps a consequence of the smaller fraction of accreted systems in this grouping compared to the YH GCs.

We have shown that the YH GC population at galactocentric distances exceeding $10 \mathrm{kpc}$ exhibits the same orientation (within uncertainties) as that defined by the MW satellites. This argues for a strong causal link between the origins of the outer halo GCs and the MW satellites. In the next section we discuss the origins of the planar arrangements of dwarf galaxies around the MW and M31, and by direct association, the origin of the outer halo GCs. 

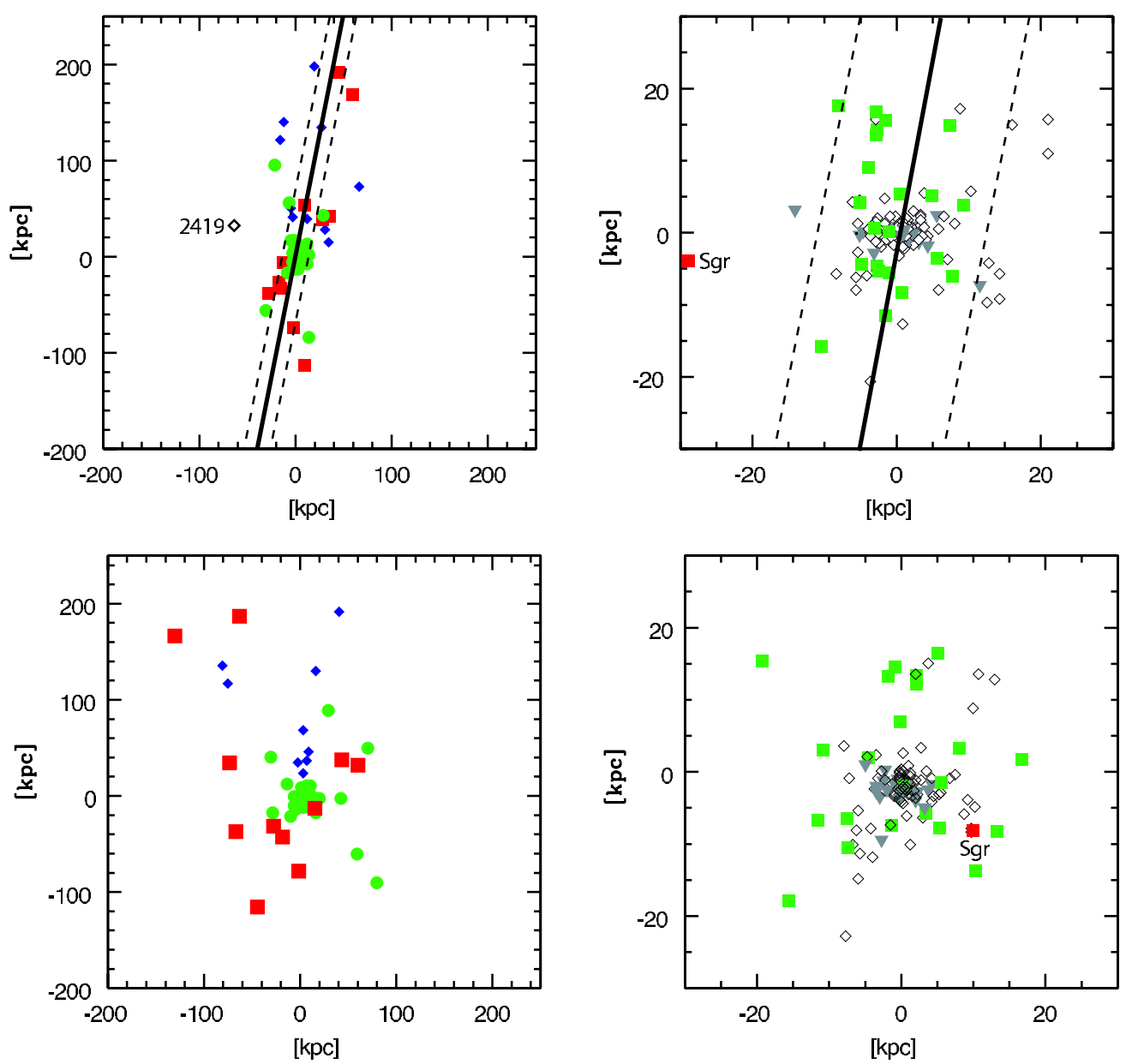

Figure 4. Top left: the PoS described by the young halo GC population of galactocentric distance greater than $10 \mathrm{kpc}$ (see Table 1) as seen edge-on to the plane (solid line, surrounded by dashed lines showing the rms thickness, viewed from $\left.\ell, b=\left(160^{\circ}, 0^{\circ}\right)\right)$. The symbols are colored the same as in Figure 3 . Top right: a zoom in on the inner halo, showing in green the YH clusters, as solid triangles the disk-bulge GCs, and as open diamonds the old halo GCs. Bottom left and right: the PoS seen face-on (viewed from $\ell, b=\left(50^{\circ}, 0^{\circ}\right)$ ).

(A color version of this figure is available in the online journal.)

\section{THE SATELLITES OF THE MILKY WAY AND M31: COSMOLOGICAL CONTEXT}

Three scenarios are presented in the literature regarding the development of the spatial distribution of satellites: that the satellites are in-situ tracers of a prolate DM halo; that they are remnants from the break up of a large progenitor at early times (i.e., the satellites are tidally disrupted galaxies); or they are tracers of collapse along filaments of LSS. Cosmological simulations of DM halos shows that under $\Lambda$ CDM cosmology halos are predominantly prolate (Libeskind et al. 2005; Zentner et al. 2005); however, the degree to which the DM halo would have to be prolate to accommodate the observed anisotropy around the MW and M31 would be extremely atypical (Kroupa et al. 2010). Furthermore, the debris stream of the disrupting Sagittarius dwarf cannot be modeled under the influence of such an extremely prolate DM halo (Law et al. 2009; Prior et al. 2009; Keller et al. 2008, 2009).

Li \& Helmi (2008) present a scenario in which all satellites fall in to the MW halo in 1-2 groups and demonstrate that such an accretion history could account for the current satellite anisotropy. D'Onghia \& Lake (2008) develop a similar scenario, reminiscent of the groups of MW satellite galaxies of LyndenBell $(1976,1982)$ and Kunkel \& Demers (1976), in which the groups formed in LMC-like DM halos. However, such a scenario has a number of problematic features as discussed in Kroupa et al. (2010): the frequency of such groups in the local volume is low; the group would have been comprised predominantly of dwarf spheroidal galaxies and this does not match to the morphology-density relation; and the group identity of satellites after infall is expected to be short-lived (Klimentowski et al. 2009).

Metz et al. (2007) present a scenario in which the satellites arise from the tidal fragmentation of a large galaxy at an early epoch. Under this scenario, the kinematics of the tidally disrupted galaxies is retained from formation as a rotationally supported disk. Metz et al. (2008, 2009b) confirm this: the inferred angular momentum vectors for the majority of classical MW satellites are directed within a $30^{\circ}$ region on the sky.

Libeskind et al. (2005, 2011) present cosmological simulations that show that substructures stream through LSS filaments that feed MW-sized halos. This accretion occurs from preferred directions on the sky and is coherent on scales from $1 \mathrm{Mpc}$ to $20 \mathrm{kpc}$. From simulated systems that contain at least 11 luminous satellites (i.e., comparable to the MW system), Libeskind et al. (2011) find 30\% possess satellites with orbital angular momenta aligned to a similar degree as the MW system. Lovell et al. (2011) find a similar result, namely that quasi-planar 


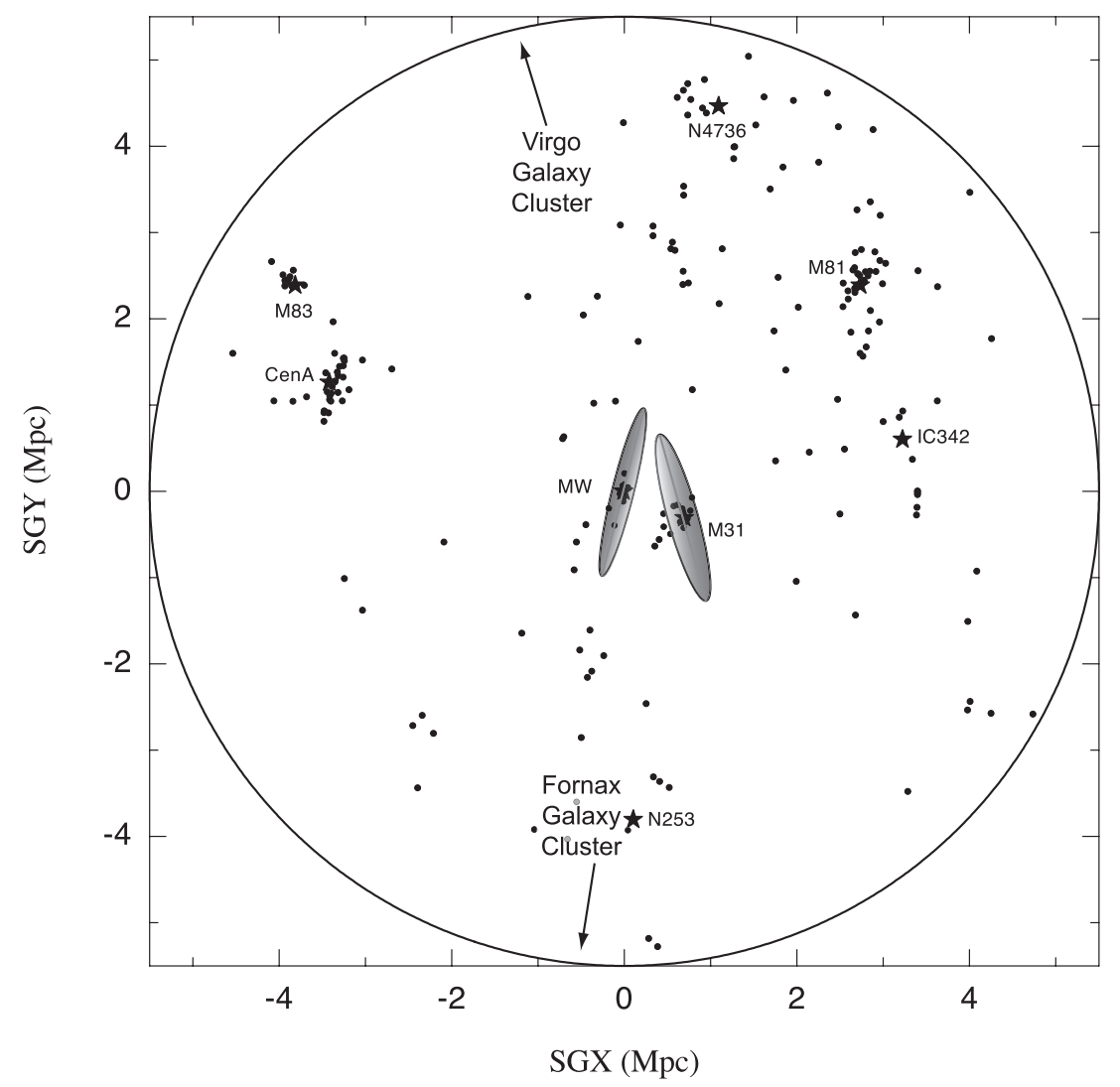

Figure 5. PoS of the Milky Way and M31 seen in the context of the supergalactic coordinate frame of reference. The shading of the ellipses represents orientation, darker is closer. In this $X-Y$ projection the PoS of both galaxies are nearly side-on and generally directed along an axis joining nearby major mass concentrations in the form of the Virgo and Fornax galaxy clusters (15 and $12 \mathrm{Mpc}$ distant from the Milky Way, respectively).

distributions of coherently rotating satellites arise naturally in the Aquarius simulations as subhalos fall in along the central spines of LSS filaments. ${ }^{3}$

In Figures 5 and 6 we show the orientation of the MW and M31 PoS in supergalactic coordinates (see de Vaucouleurs et al. 1991 for definition). The supergalactic plane is defined by the distribution of galaxies $\sim$ few Mpc about the MW. Within this volume, galaxies are confined to a $\sim 2 \mathrm{Mpc}$ thick plane (seen side on in Figure 6). Seen from above (Figure 5) the MW and M31 PoS are both highly inclined with respect to the supergalactic plane. As discussed in Metz et al. (2007) there is no apparent spatial connection between the MW and M31 PoS as the M31 PoS is inclined at $\sim 55^{\circ}$ to the MW PoS. It is, however, notable that the PoS of both galaxies is well aligned with an axis between the two major mass distributions within $20 \mathrm{Mpc}$ : the Virgo and Fornax clusters (Karachentsev et al. 2003). This was remarked upon by Navarro et al. (2004) in their study of the galaxies of the local supergalactic plane. ${ }^{4}$ Here they found that not only are the satellites of nearby early-type galaxies preferentially confined to polar planes relative to their disks but that the galaxy disk is orientated along the major axis of surrounding LSS material. This is also the case for the MW

\footnotetext{
3 However, we note that Kroupa et al. (2010) argue this is a selection effect citing that the MW-like galaxies with at least 11 luminous satellites account for $1.4 \%$ of simulated galaxies in Libeskind et al. (2011) and hence that only $0.4 \%$ (i.e., $30 \%$ of $1.4 \%$ ) of all existing MW-like $\Lambda$ CDM halos would host an MW-type galaxy with an appropriate satellite spatial distribution.

4 However, we note that Yang et al. (2006) and Agustsson \& Brainerd (2006) find that this alignment does not appear to extend to scales less than the typical virial radius.
}

and M31, where, as seen in Figures 5 and 6, the PoS are highly inclined to the stellar disks of their hosts.

\section{DISCUSSION: THE ORIGIN OF OUTER HALO GLOBULAR CLUSTERS}

It is our thesis that the observed affinity between the planar distribution of outer halo GCs and that of the MW satellites implies a common origin. In this scenario, the outer halo GCs are accreted to the MW as part of galaxies from the surrounding LSS. They enter as entities residing in DM subhalos in LSS filaments that stream into the MW DM halo. Upon entry, potential subhalos may deliver the GCs from their natal DM subhalos via tidal disruption and dispersal of dwarf galaxies (akin to the simulations of Libeskind et al. 2011) or from the early tidal disruption of a moderate mass galaxy (like that envisaged by Kroupa et al. 2010). We discount the possibility of "free-floating" GCs as this would imply $\mathrm{DM}$ dominance and in the case of at least one of the outer halo GCs (Pal 14; Jordi et al. 2009) there is no evidence for DM.

We see in the spatial distribution of the outer halo GCs evidence of their accretion origin. This is a direct observation of the importance of accretion to galaxy formation as inferred from the properties of the MW GC system in the seminal work of Searle \& Zinn (1978). The only direct observations of this process of GC accretion are in the disrupting Sagittarius dwarf (delivering at least five GCs into the MW halo; Da Costa \& Armandroff 1995; Martínez-Delgado et al. 2002; Law \& Majewski 2010), perhaps in the case of the putative Canis Major dwarf (Martin et al. 2004) and in the GCs of M31 associated 


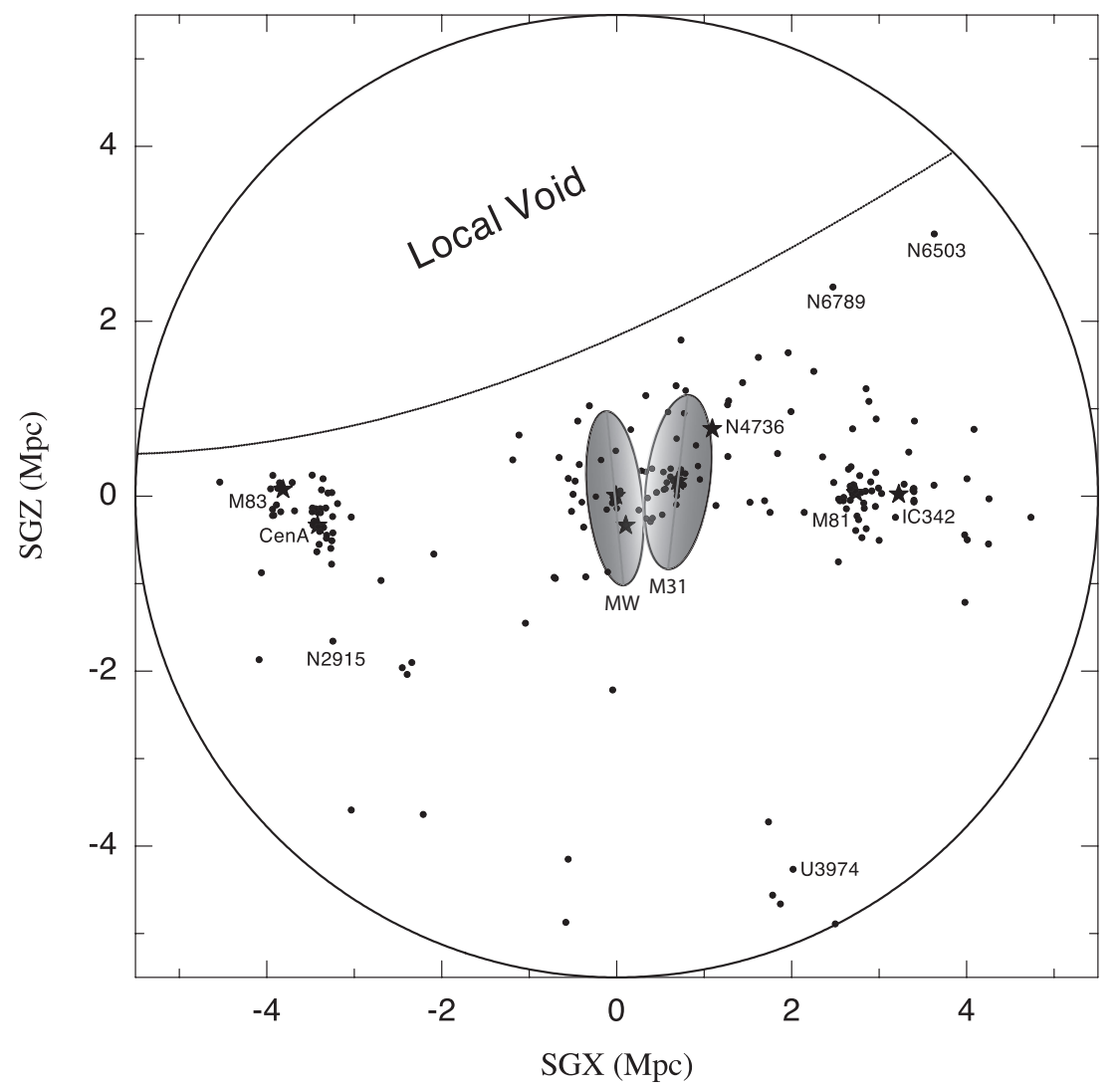

Figure 6. As in Figure 5 but in the $X-Z$ section through the supergalactic plane.

with tidal stellar streams in the vicinity of M31 (Mackey et al. 2010).

Much corroborating evidence is presented for this conclusion is presented in the literature. A particularly distinctive feature of the YH GCs is their distribution of core radii (Mackey \& Gilmore 2004; see also Mackey \& van den Bergh 2005). The core radii $\left(r_{c}\right)$ of the YH GCs show a very long tail to very large radii (all but one GC with $r_{c}>9$ pc resides in the Mackey \& Gilmore grouping of GCs associated with the outer halo GCs). Furthermore, the $r_{c}$ distribution of these GCs shows no statistical difference to that observed from a compilation of GCs from the LMC, Fornax and Sagittarius dwarf galaxies (Mackey $\&$ Gilmore 2003). Together with the similarity in morphology of the HB between the external GCs and the YH GC grouping, this evidence leads Mackey \& Gilmore (2004) to propose that all the YH GCs are accreted.

The implications of the size distribution for an accreted origin of the outer halo GCs are elucidated in the study of Hurley \& Mackey (2010). In this study, the authors use $N$-body simulations of star clusters in a tidal field to investigate the conditions required to produce and sustain a GC of large core radius. Hurley \& Mackey show that clusters may be born with a range of $r_{c}$ governed by how much the cluster fills its initial tidal radius. When a cluster completely fills its natal tidal radius an extended GC can result $\left(r_{c}>10 \mathrm{pc}\right)$. Conditions for a cluster to completely fill its tidal radius at birth are optimal in regions where background tidal forces are low: such conditions are best satisfied at large distances from MW-like galaxies and in dwarf systems (see discussion in Elmegreen 2008 and Da Costa et al. 2009). This again argues for the accretion of the outer halo GCs to the MW.
The timing of the delivery of the outer YH GCs through accretion is not constrained by the physical parameters of the GCs. Gnedin (1997) describes the stability of the MW's GCs against two-body relaxation, tidal truncation, and tidal shocks due to passage through the disk and due to close proximity to the bulge. With the exception of two clusters (Pal 1 and Pal 13), GCs of $\mathrm{R}>10 \mathrm{kpc}$ are expected to be long-lived, with lifetimes of between 5 and 100 Hubble times. Hence, by this criterion, these systems could have been accreted into the MW at any stage over the last Hubble time. We can ask the question: how many satellites of a given mass are required to contribute the observed (conservative) $22 \mathrm{YH} \mathrm{GCs}$ at R $>10 \mathrm{kpc}$ : Given the specific frequency of GCs $\left(S_{N}\right)$ as a function of host galaxy luminosity (first introduced by Harris \& van den Bergh 1981, and, e.g., discussed recently by Georgiev et al. 2010), we find this would require, for example, approximately 2 Magellaniclike $\left(M_{V} \sim-18\right.$ with GC $\left.S_{N} \sim 1\right)$ systems or 22 systems with Sculptor-like luminosities $\left(M_{V} \sim-11\right.$ with GC $\left.S_{N} \sim 70\right)$. If we then consider that the number of accreted clusters is likely to be supplemented by 10-12 OH clusters (Mackey \& Gilmore 2004), we estimate that the MW may have experienced mergers with three Magellanic-like to 30 Sculptor-like luminosity systems over the MW's lifetime. ${ }^{5}$ Such deposition would contribute around half of the mass of the stellar halo. In order to contribute stars that are of sufficiently low metallicity (and sufficiently high $[\alpha / \mathrm{Fe}])$ to match those found in the halo, such accretion must have taken place early on in the chemical evolution of the accreted galaxies.

\footnotetext{
5 When considering the number of mergers, it should be noted that there is almost a factor of 10 variation exhibited in the $S_{N}$ at given galaxy luminosity.
} 


\section{CONCLUSIONS}

In this study we have partitioned the MW's GC system into groups according to metallicity, HB morphology, and the stellar pulsation properties of their RR Lyrae population. The grouping broadly align with relative ages of the constituent clusters. When partitioned in this way the MW's YH GCs (of galactocentric radius greater than $10 \mathrm{kpc}$ ) are anisotropically spatially distributed and the distribution can be described by a plane $24 \pm 4 \mathrm{kpc}$ in thickness (rms). The normal to this plane is directed toward $(\ell, b)=\left(156^{\circ} \pm 6^{\circ},-8^{\circ} \pm 5^{\circ}\right)$. In contrast, the $\mathrm{OH}$ and $\mathrm{DB}$ groups are consistent with an isotropic spatial distribution. The plane defined by the $\mathrm{YH}$ population is statistically indistinguishable from that described by the satellite galaxies of the MW $\left(\ell, b=166^{\circ} \pm 4^{\circ},-13^{\circ} \pm 3^{\circ}\right)$. We argue therefore that the YH GCs share the same origin as that of the system of satellite galaxies about the MW.

Simulations of the satellite galaxies of MW-like systems have shown that the planar arrangement of satellites arises naturally from accretion of subhalos along filaments of the surrounding LSS which are directed toward the host halo. Once accreted, the GCs of the subhalos are delivered to the MW halo by tidal disruption.

The finding of a common spatial anisotropy amongst the MW satellites and the YH GCs is an observation of the accretion origin of the outer GCs. It is a direct confirmation of numerous lines of evidence that have shown the outer halo GCs to possess individual properties that are shared most closely with those found in the GC systems of low-mass galaxies.

This paper arose from discussions at the Galactic Studies with the LAMOST Surveys workshop held at the Kavli Institute for Astronomy at Peking University, 2010 July. S.C.K. and D.M. would like to express their gratitude to the organizers for providing a fruitful meeting. S.C.K. and G.S.D.C's research has been supported in part by the Australian Research Council through Discovery Project Grant DP0878137. D.M. acknowledges the financial support from an Australian Research Fellowship (DP1093431) from the Australian Research Council.

\section{REFERENCES}

Agustsson, I., \& Brainerd, T. G. 2006, ApJ, 644, L25

Agustsson, I., \& Brainerd, T. G. 2010, ApJ, 709, 1321

Bailin, J., Power, C., Norberg, P., Zaritsky, D., \& Gibson, B. K. 2008, MNRAS, 390, 1133

Besla, G., Kallivayalil, N., Hernquist, L., et al. 2010, ApJ, 721, L97

Brainerd, T. G. 2005, ApJ, 628, L101

Catelan, M. 2009, Ap\&SS, 320, 261

Cohen, J. G., Kirby, E. N., Simon, J. D., \& Geha, M. 2010, ApJ, 725, 288

Da Costa, G. S., \& Armandroff, T. E. 1995, AJ, 109, 2533

Da Costa, G. S., Grebel, E. K., Jerjen, H., Rejkuba, M., \& Sharina, M. E. 2009, AJ, 137, 4361

de Vaucouleurs, G., de Vaucouleurs, A., \& Corwin, H. G., Jr., et al. (ed.) 1991, Third Reference Catalogue of Bright Galaxies (New York: Springer)
D’Onghia, E., \& Lake, G. 2008, ApJ, 686, L61

Dotter, A., Sarajedini, A., \& Anderson, J. 2011, ApJ, 738, 74

Dotter, A., Sarajedini, A., Anderson, J., et al. 2010, ApJ, 708, 698

Eggen, O. J., Lynden-Bell, D., \& Sandage, A. R. 1962, ApJ, 136, 748

Elmegreen, B. G. 2008, ApJ, 672, 1006

Fisher, N. I., Lewis, T., \& Embleton, B. J. 1987, Statistical Analysis of Spherical Data (Cambridge: Cambridge Univ. Press)

Forbes, D. A., \& Bridges, T. 2010, MNRAS, 404, 1203

Georgiev, I. Y., Puzia, T. H., Goudfrooij, P., \& Hilker, M. 2010, MNRAS, 406, 1967

Gnedin, O. Y. 1997, ApJ, 487, 663

Harris, W. E. 1996, AJ, 112, 1487

Harris, W. E., \& van den Bergh, S. 1981, AJ, 86, 1627

Holmberg, E. 1969, Ark. Astron., 5, 305

Hurley, J. R., \& Mackey, A. D. 2010, MNRAS, 408, 2353

Jordi, K., Grebel, E. K., Hilker, M., et al. 2009, AJ, 137, 4586

Karachentsev, I. D., Makarov, D. I., Sharina, M. E., et al. 2003, A\&A, 398, 479

Keller, S. C., Da Costa, G. S., \& Prior, S. L. 2009, MNRAS, 394, 1045

Keller, S. C., Murphy, S., Prior, S., Da Costa, G. S., \& Schmidt, B. 2008, ApJ, 678,851

Keller, S. C., Schmidt, B. P., Bessell, M. S., et al. 2007, PASA, 24, 1

Klimentowski, J., Łokas, E. L., Kazantzidis, S., et al. 2009, MNRAS, 400, 2162

Koch, A., \& Grebel, E. K. 2006, AJ, 131, 1405

Kroupa, P., Famaey, B., de Boer, K. S., et al. 2010, A\&A, 523, 32

Kroupa, P., Theis, C., \& Boily, C. M. 2005, A\&A, 431, 517

Kunkel, W. E., \& Demers, S. 1976, in Royal Greenwich Observatory Bulletin,

Vol. 182, The Galaxy and the Local Group, ed. R. J. Dickens, J. E. Perry, F.

G. Smith, \& I. R. King (Herstmonceux: The Observatory), 241

Law, D. R., \& Majewski, S. R. 2010, ApJ, 718, 1128

Law, D. R., Majewski, S. R., \& Johnston, K. V. 2009, ApJ, 703, L67

Li, Y., \& Helmi, A. 2008, MNRAS, 385, 1365

Libeskind, N. I., Frenk, C. S., Cole, S., et al. 2005, MNRAS, 363, 146

Libeskind, N. I., Knebe, A., Hoffman, Y., et al. 2011, MNRAS, 411, 1525

Lovell, M. R., Eke, C. R., Frenk, C., \& Jenkins, A. 2011, MNRAS, 413, 3013

Lynden-Bell, D. 1976, MNRAS, 174, 695

Lynden-Bell, D. 1982, Observatory, 102, 7

Mackey, A. D., \& Gilmore, G. F. 2003, MNRAS, 340, 175

Mackey, A. D., \& Gilmore, G. F. 2004, MNRAS, 355, 504

Mackey, A. D., Huxor, A. P., Ferguson, A. M. N., et al. 2010, ApJ, 717, L11

Mackey, A. D., \& van den Bergh, S. 2005, MNRAS, 360, 631

Marín-Franch, A., Aparicio, A., Piotto, G., et al. 2009, ApJ, 694, 1498

Martin, N. F., Ibata, R. A., Bellazzini, M., et al. 2004, MNRAS, 348, 12

Martin, N. F., McConnachie, A. W., Irwin, M., et al. 2009, ApJ, 705, 758

Martínez-Delgado, D., Zinn, R., Carrera, R., \& Gallart, C. 2002, ApJ, 573, L19

Metz, M., Kroupa, P., \& Jerjen, H. 2007, MNRAS, 374, 1125

Metz, M., Kroupa, P., \& Jerjen, H. 2009a, MNRAS, 394, 2223

Metz, M., Kroupa, P., \& Libeskind, N. I. 2008, ApJ, 680, 287

Metz, M., Kroupa, P., Theis, C., Hensler, G., \& Jerjen, H. 2009b, ApJ, 697, 269

Navarro, J. F., Abadi, M. G., \& Steinmetz, M. 2004, ApJ, 613, L41

Parmentier, G., \& Grebel, E. K. 2005, MNRAS, 359, 615

Prior, S. L., Da Costa, G. S., \& Keller, S. C. 2009, ApJ, 704, 1327

Rey, S.-C., Yoon, S.-J., Lee, Y.-W., Chaboyer, B., \& Sarajedini, A. 2001, AJ, 122,3219

Richardson, J. C., Irwin, M. J., McConnachie, A. W., et al. 2011, ApJ, 732, 76

Sales, L., \& Lambas, D. G. 2009, MNRAS, 395, 1184

Searle, L., \& Zinn, R. 1978, ApJ, 225, 357

Yang, X., van den Bosch, F. C., Mo, H. J., et al. 2006, MNRAS, 369, 1293

Yoon, S.-J., \& Lee, Y.-W. 2002, Science, 297, 578

Zentner, A. R., Kravtsov, A. V., Gnedin, O. Y., \& Klypin, A. A. 2005, ApJ, 629, 219

Zinn, R. 1985, ApJ, 293, 424

Zinn, R. 1993, in ASP Conf. Ser. 48, The Globular Cluster-Galaxy Connection, ed. G. H. Smith \& J. P. Brodie (San Francisco, CA: ASP), 38 